\title{
An unusual case of ductal carcinoma in situ
}

\section{Abstract}

Ductal carcinoma in situ (DCIS; intraductal carcinoma) is a noninvasive breast cancer originating from the cells that line the mammary ducts. Patients with DCIS can be asymptomatic at the time of presentation (radiographic findings on mammogram) or present with symptoms such as a palpable mass or nipple discharge. ${ }^{1}$ We present an unusual case of DCIS in a 40 year old woman with recent palpable mass. After diagnostic mammography and breast ultrasound, BIRADS 4c category was assigned and biopsy was performed. Pathology report indicates the presence of two histollogical types: DCIS and also invasive lobulillar carcinoma.

\author{
Volume 8 Issue 4 - 2017
}

Cuituny Romero AK, Onofre Castillo JJ, Putz Botello MD, Decanini Arcaute H, Santana Vela IA,Torres Gomez E

Radiology Departement, Christus Muguerza High Specialty Hospital, Monterrey University, Mexico

Correspondence: Cuituny Romero AK, Radiologist, Radiology department of Christus Muguerza High Specialty Hospital and Monterrey University, Hidalgo 2525 Monterrey, Nuevo Leon, Mexico,Tel8116117196,Email cuituny@gmail.com

Received: July 27, 2017 | Published: August 30, 2017
Abbreviations: DCIS, ductal carcinoma in situ; CC, craniocaudal; MLO, mediolateral oblique; US, ultrasound; DLU, terminal ductal lobular unit; ADH, atypical ductal hiperplasia; LCIS, lobular carcinoma in situ

\section{Introduction}

Ductal carcinoma in situ is defined by the proliferation of tumor cells within the terminal lobular unit with preservation of the basement membrane. ${ }^{2}$ Before screening mammography, DCIS accounted only the 0.8 to $5 \%$ of all breast cancers. ${ }^{2}$ Currently it represents $25 \%$ of all cancers in the US. ${ }^{2}$ It was first recognized in the early 20th century, mainly in mammography specimens with invasive malignancy. ${ }^{2}$ Studies indicate that it is a precursor of invasive carcinoma in an average time of 5 to 8 years. ${ }^{2} 25-50 \%$ of women who have untreated DCIS will develop invasive cancer in the same quadrant of the breast. If ductal carcinoma in situ is not completely resected, $33 \%$ will recur and $50 \%$ of these will be invasive carcinoma. ${ }^{2}$ The American College of Radiology recommends annual screening mammography

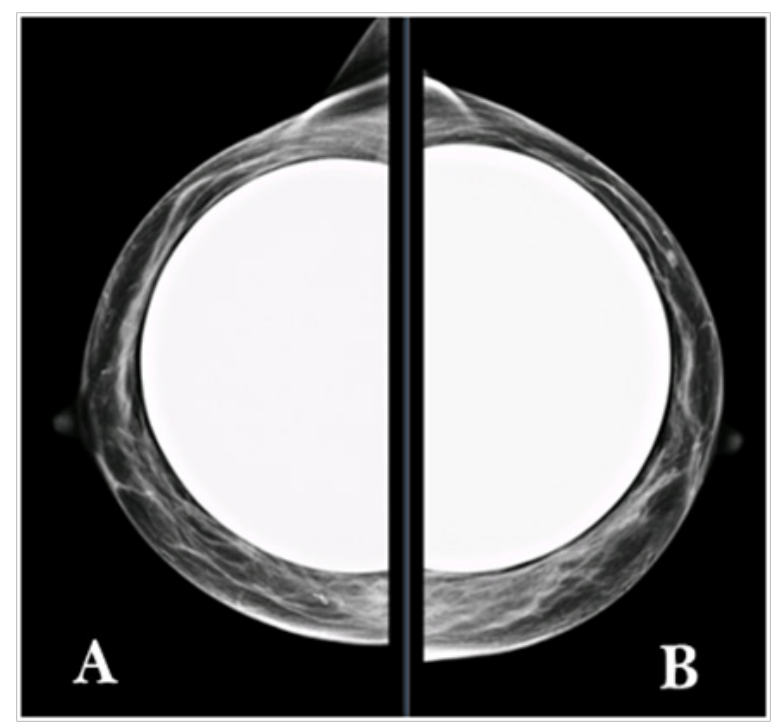

Figure I a) Right breast. b) Left breast. CC mammography views shows moderately dense fibroglandular parenchyma (pattern b ACR). for women starting at age 40. Breast cancer incidence increases substantially around age 40 and even earlier for high risk women and women of color. ${ }^{3}$

\section{Case presentation}

40 years old female with an antecedent of cosmetic surgery with placement of breast implants 10 years ago. Her current condition is recent palpable area on right breast. Mammongraphy and ultrasound were performed on January 8, 2015 (Figure 1-10).

Left breast without findings of interest on mammography and ultrasound. The palpable finding of right breast has an indeterminate appearance. Classifying BI-RADS category 4c: finding with high suspicion of malignancy. After this diagnosis, US guided needle biopsy of right mammary gland nodule showed intermediate grade in situ carcinoma with comedonecrosis. Immunohistochemistry demonstrated positivity for P63 and CD10 in the myothelial cells surrounding ducts showing carcinoma in situ (Figure 11-15).

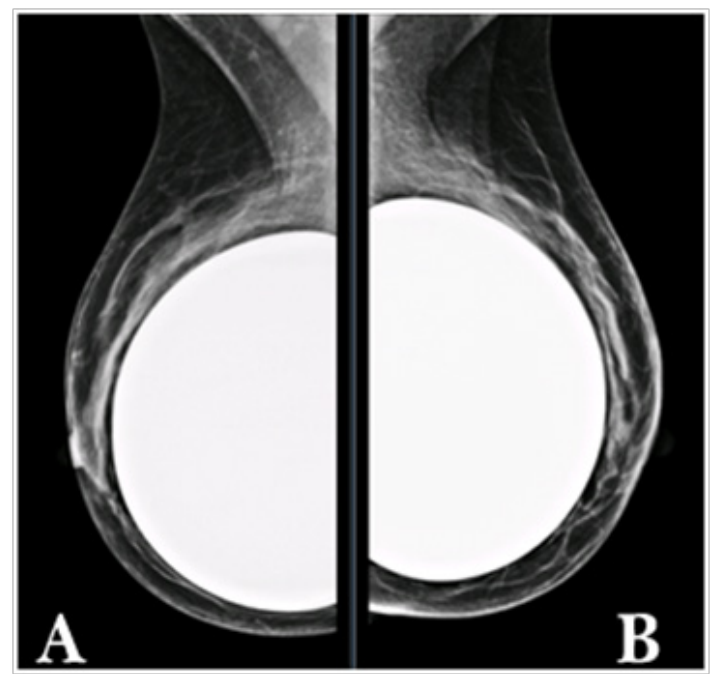

Figure 2 a) Right breast. b) Left breast. MLO views shows moderately dense fibroglandular parenchyma (pattern b ACR) with predominance in the upper quadrants. Retropectoral breast implants with preserved morphology. 


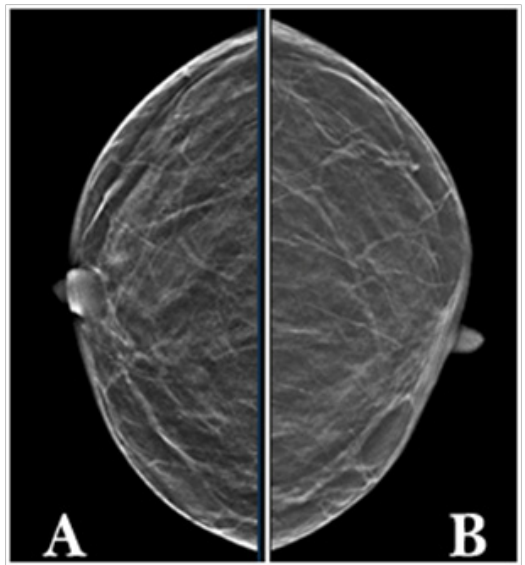

Figure 3 a) Right breast. b) Left breast. CC Eklund views shows no abnormalities.

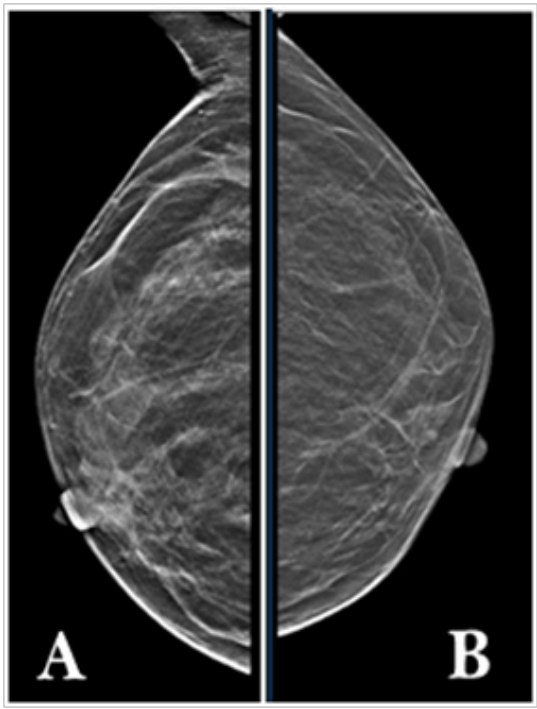

Figure 4 a) Right breast. b)Left breast. MLO Eklund views with no abnormalities.

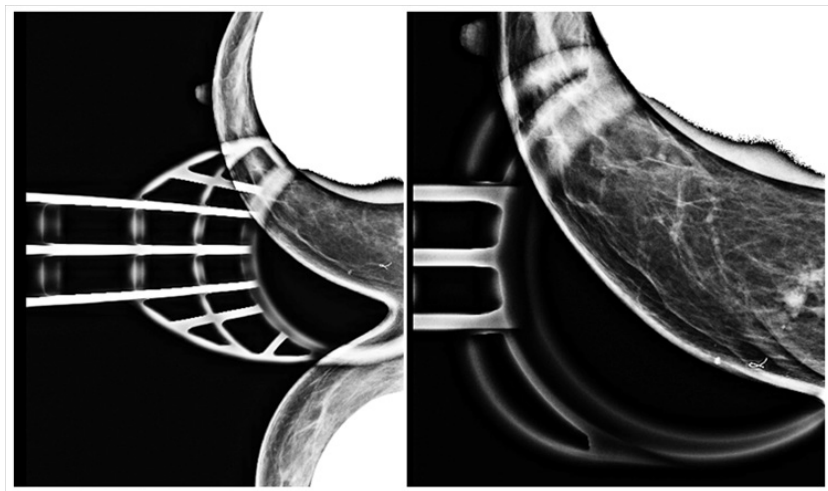

Cleavage view

Right breast magnification cleavage view

Figure 5 \& 6 Rigth breast special views. In the internal upper quadrant of the right breast, there is an asymmetry located at $10 \mathrm{~cm}$ from the nipple, evident in special views. It is nodular, irregular with spiculate and dense contours, occupying an area of $1.8 \mathrm{~cm}$. It is associated with distortion and some punctiform calcifications. Adjacent tissue shows a diffuse increase in density (suggestive of interstitial edema). These findings represent the palpable area.

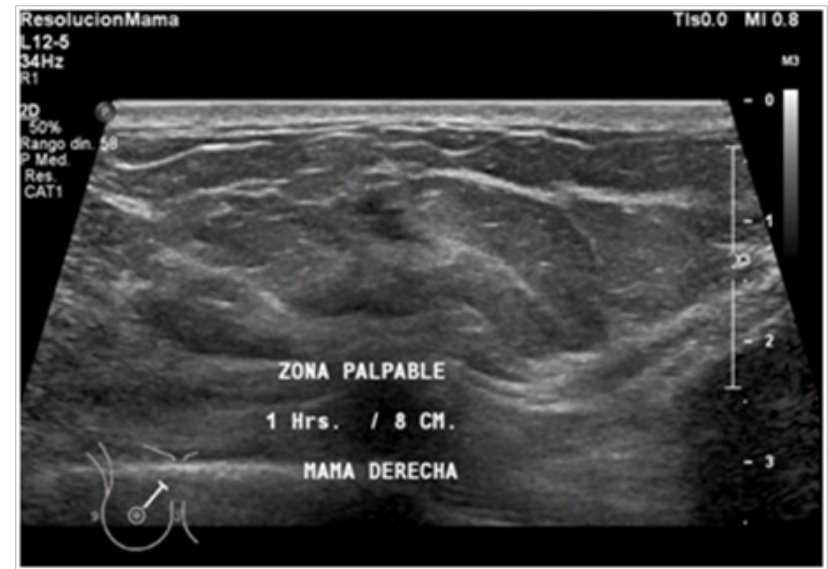

Figure 7 Right breast ultrasound was performed observing in the upper internal quadrant at I o'clock $/ 8 \mathrm{~cm}$ from the nipple, an irregular solid nodule with spiculated and poorly circumscribed margins. Adjacent tissue shows diffuse increase in echogenicity (suggestive of interstitial edema). These findings represent the palpable area.

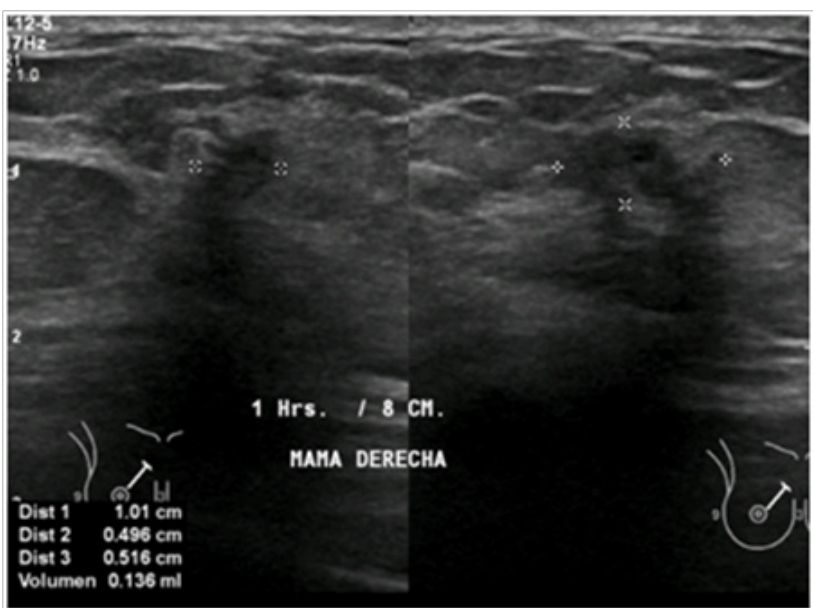

Figure 8 Right breast ultrasound show irregular nodule that measures $1.0 \times$ $0.5 \times 0.5 \mathrm{~cm}$, with heterogeneous echotexture and moderately hypoechoic. It is associated with calcification and echogenic halo.

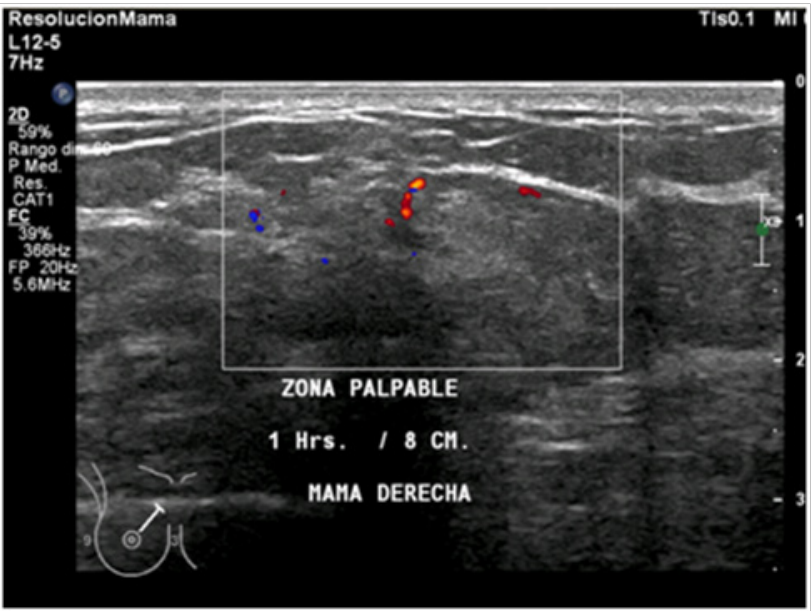

Figure 9 Right breast ultrasound with color doppler shows the presence of central and peripheral vascularity into the nodule. 


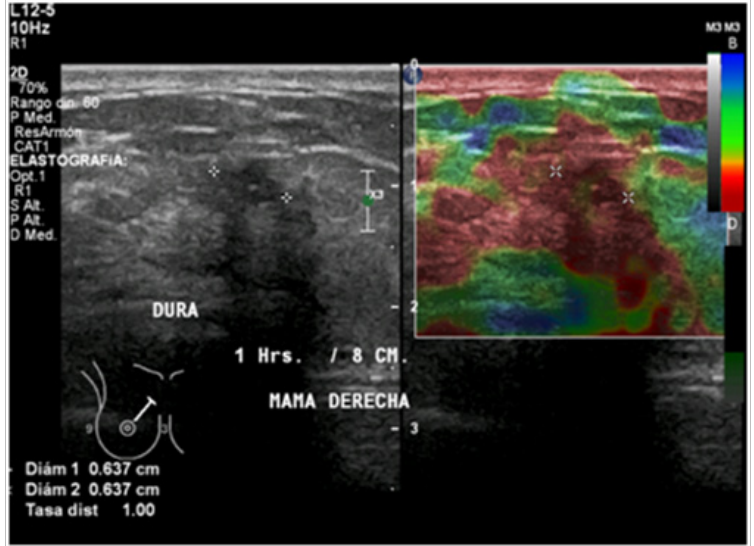

Figure I 0 Right breast ultrasound with elastography shows hard consistency into the nodule, including areas of adjacent tissue.
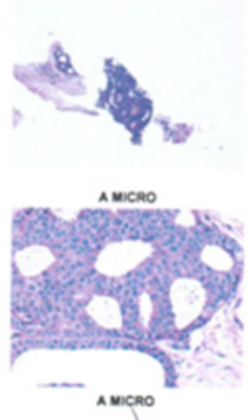

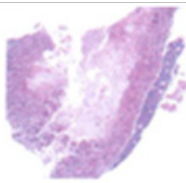

A wicko

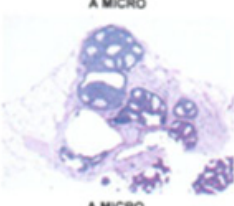

A wiceo

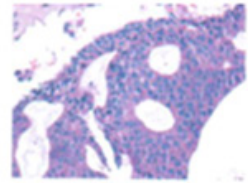

A wocko

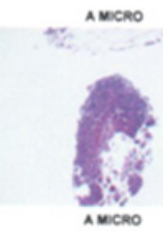

Figure II Fragments of right mammary gland were observed with few glandular units alternating with broad ducts showing an intermediate grade carcinoma in situ with cells that completely fill the lights and show their characteristic arches, the nuclei are polymorphic, molded and hyperchromatic and with abnormal mitosis. Tubeles with comedogenic pattern.

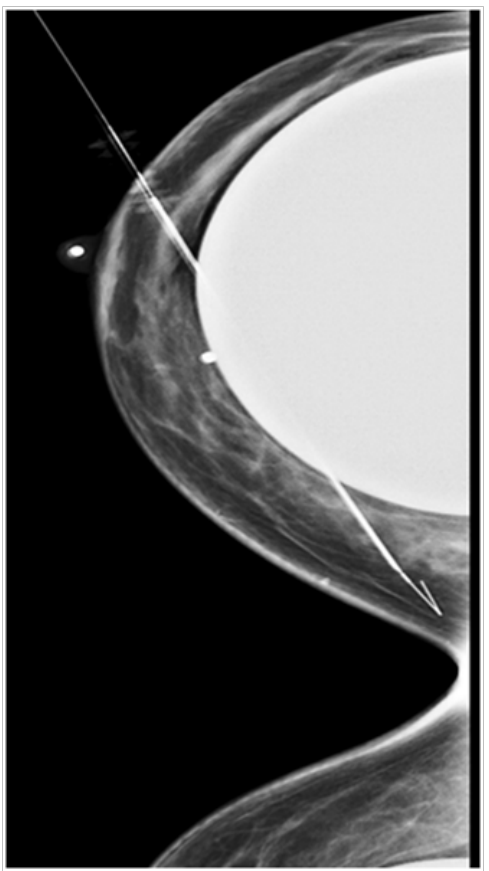

Figure I 2 Right breast cleavage view marking with mammography before surgical excision.
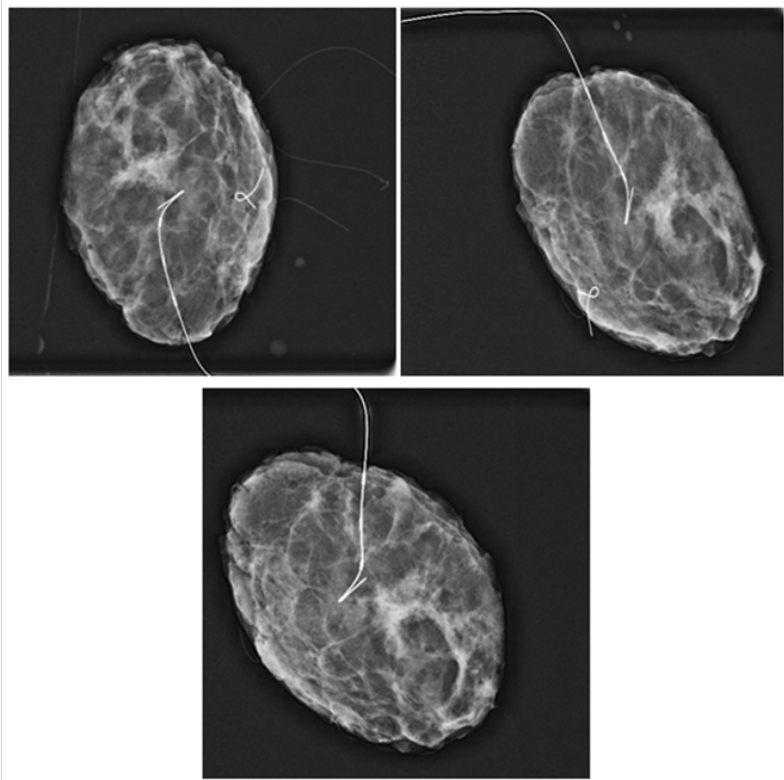

Figure I 3, I 4 \& I 5 Right breast postoperative pieces. Identifying an irregular and hypoechoic solid nodule measuring $1.0 \mathrm{~cm}$, is associated with spicules, calcifications and distortion of the adjacent tissue.

\section{Pathology report}

a. Procedure: Excision with harpoon marking.

b. Lymph node resection: No

c. Tumor side: Right breast.

d. Tumor site: Internal superior quadrant.

e. Tumor size: $3.3 \mathrm{cms}$

1. Histological type: Ductal carcinoma in situ with microinvasion focuss.

a. Growth patterns: Cribiform and comedonic.

b. Nuclear grade: High.

c. Necrosis: Present (comedonic pattern).

2. Histological type: Invasive lobular carcinoma.

a. Size of the tumor: $0.5 \mathrm{cms}$.

b. Focality: Unifocal.

c. Associated ductal carcinoma: Present.

d. Angioinvasion: Not located.

e. Perineural invasion: Not identified.

Limits of surgical section.

Internal (inked in blue) positive for infiltrant lobular carcinoma.

Superior internal angle (in-yellow painting) positive for carcinoma in situ.

Lower angle (inked in red) free of injury.

Carcinoma in situ. Internal limit (into in green) with lesion carcinoma in situ at $0.2 \mathrm{cms}$ from the ink. 
Surgical deep limit (inked in black at $0.5 \mathrm{cms}$ of injury (carcinoma in situ).

\section{Tnm classification}

$$
\begin{aligned}
& \text { I. pTis (DCIS) } \\
& \text { II. pT1b: N: NX, MX. }
\end{aligned}
$$

\section{Case discussion}

The ductal system begins at the nipple. Between 25-30 galactophore ducts extend from the nipple and branch into segmental, subsegmental and terminal ducts. The terminal ducts are associated with lobules and form the terminal lobular ductal unit. In the normal breast, the terminal ducts and lobes are lined with a layer of epithelium and an outer layer of myoepithelium. Most breast malignancies arise within the TDLU. ${ }^{4}$

When the TDLU epithelium proliferates and there is more than one layer of cells it is called atypical ductal hyperplasia. If multiple cell lines proliferate or are cloned asymmetrically, the terminal duct or the lobule is filled with a heterogeneous population of small cells. This is called typical or benign hyperplasia. If the proliferating cells are uniform in appearance, the lesion is classified as atypical cell hyperplasia or DCIS grade 1 (low grade). The tumor is labeled as $\mathrm{ADH}$ if the abnormal proliferation is very small involving no more than two TDLU's or $2 \mathrm{~mm}$. If the tumor exceeds this size, it is labeled as DCIS. ${ }^{4}$

\section{A. Important histopatholigical prognostic characteristics. ${ }^{4}$}

a. Nuclear grade. It is the most important.

i. Low-grade lesions usually have positive estrogen receptors, have small nuclei, and few mitoses.

ii. The intermediate grade moderately enlarged and heterogeneous nuclei with few mitoses.

iii. High grade, with estrogen receptor negative. Aneuploid nuclei with high mitosis rate which is irregular.

b. DCIS may have several cell degrees, it is labeled by the one that predominates.

c. Necrosis. The second most important feature: the absence or presence of central necrosis in the duct. Rate of recurrence is higher with central necrosis. A DCIS study reported a recurrence rate of $19 \%$ with central necrosis, $10 \%$ for cribiform without necrosis and $0 \%$ micropapillary without necrosis.

d. Cellular phenotype: Apocrine, Endocrine, Clear cells, Seal ring.

e. Pattern of growth: Micropapillary, Cribiform, Solid, Climbing plant.

f. Architectural Pattern: Solid, Cribiform, Papillary, Micropapillary, Comedogenic.

B. Appearance of DCIS by mammography. ${ }^{4}$

a. Calcifications. $62-72 \%$ as grouped calcifications.

b. $12-30 \%$ calcifications in the parenchyma or into a circumscribed mass.

c. $0-12 \%$ as a mass without calcifications.

d. Rare: distortions in the architecture, dilated ducts (subareolar or distal to the nipple).

e. $6-15 \%$ is not detected by mammography, incidental finding.
Although the finding of clustered calcifications on mammography is extremely sensitive for the diagnosis of DCIS, the specificity of this finding is only $10 \%$ to $35 \%$, as a result of this relatively low specificity about $60-80 \%$ of biopsies by calcifications is associated with benign histological findings. ${ }^{4}$ DCIS and contralateral malignancy: 2.2 to $22 \%$ (DCIS or invasive cancer). ${ }^{4}$ Multicentricity in $12-80 \%$. Defined by the presence of tumor foci in non-contiguous quadrants or at least $5 \mathrm{~cm} .{ }^{4}$ Multifocality is defined as the presence of more than one lesion in a single quadrant or within a radius of $5 \mathrm{~cm} .{ }^{4}$ Hollland and colleagues, demonstrated that DCIS spreads contiguously in the same ductal or adjacent system. ${ }^{4}$

Studies have investigated the utility of pathologic grading of DCIS for predicting the risk of recurrence after conservation therapy. The simplest, most reproducible classification system is the Van Nuys system, which identifies three groups of DCIS lesions. In the Van Nuys system, lesions are differentiated first according to nuclear grade (high grade or other grade) and then according to whether necrosis is present or absent. ${ }^{5}$ The Van Nuys Prognostic Index, adopted from a review in which a risk category was developed based on margin status, histologic subtype, tumor size, and patient age using a cohort of DCIS patients treated from 2 institutions, continues to be used by some practitioners as part of their decision-making process for adjuvant radiation after local excision. It is important to note that the data from this "scoring system" were derived from retrospective data and that all randomized prospective data published to date have consistently demonstrated an improvement in local control in all patients. ${ }^{6}$

Incidence of comedonic type (aggressive) has been maintained. Incidence of noncomedonic has increased from 15 to 22 times. 68 to $92 \%$ of cases are detected as calcifications on mammography. $2-3 \%$ as an asymmetry. ${ }^{2}$ Immunohistochemistry (IHC) is used to characterize intracellular proteins or various cell surfaces in all tissues. Individual markers or more often panels of various marker proteins can be used to characterize various tumour subtypes, confirm tissue of origin, distinguish metastatic from primary tumour and provide additional information which may be important for prognosis, predicting response to therapy or evaluating residual tumour post-treatment. ${ }^{\text {? }}$

The most important diagnostic problems that occur in mammary gland tumor pathology are: the differential diagnosis of various types of benign lesions and carcinoma; differentiating between carcinoma in situ and invasive carcinoma, diagnosis and differentiation of microinvasion and its imitating lesions and confirming the breast as the primary site in metastatic carcinoma. ${ }^{7}$ p63 is a homolog of p53, it has now been shown that about $10 \%$ to $15 \%$ of invasive tumors, particularly high-grade and metaplastic carcinomas, express p63, although the staining is usually weaker than that seen in myoepithelial cells. Similarly, foci of squamous differentiation stain positively. ${ }^{7}$

CD10-positive cells have been reported in the stroma of prostate, breast, colorectal, and lung carcinomas. ${ }^{8} \mathrm{CD} 10$-cell surface zincdependent metalloproteinase, has been demonstrated on the stromal cells of some breast carcinomas, and suggested to be upregulated in breast cancer cells. Some experimental data indicate that CD10 may be a potential target for new cancer therapies, as it is involved in cleavage of doxorubicin, critical component of many cancer treatment protocols, and results in chemoresistance. Inhibition of CD10 enzymatic activity may enhance the antitumor efficacy of traditional chemotherapeutic regimens. ${ }^{8}$

\section{Conclusion}

This case illustrates the importance of performing mammography in women at age of 40 . In this particular patient, we have the antecedent 
of breast augmentation 10 years ago with no apparent control with some imaging method. If the patient also has symptoms or palpable mass, the study we will perform will be diagnostic as the patient will come for a specific problem and we will have to perform the work-up with additional mammography, ultrasound or breast MRI if necessary, in order to integrate the information and give a BIRADS category and take the appropiate measures.

This patient returned to the breast imaging deparment for a preoperative marking of the lesion in the right breast and we had the opportunity to see the postoperative pieces by image that allowed us to corroborate the findings identified in the diagnostic studies. Finally, to know that the patient not only had carcinoma in situ but also inasive lobular carcinoma which allows the clinician a more optimal approach for the appropiate management and treatment of the patient. Patients need to be informed that routine mammography should be performed at age 40 even if there are no symptoms. If the patients have a significant family, personal or surgical history, the control with imaging methods should be performed before age 40, either with mammography or ultrasonography depending on the personal clinical history in each patient, the best method for monitoring will be suggested.

American collegue of radiology (ACR) reccomends to woman with breast augmentation: ${ }^{9}$

a. Asymptomatic women with breast implants may undergo screening mammography. ${ }^{9}$

b. Facilities must have procedures in place to inquire whether patients have breast implants before a mammogram is performed. ${ }^{9}$

c. If a facility does not provide implant imaging services, it should refer the patient to other facilities that provide such services. ${ }^{9}$

\section{Acknowledgments}

None.

\section{Conflicts of interest}

Authors declare there are no conflicts of interest.

\section{References}

1. Kaufman S, Harris E, Bailey L, et al. American College of Radiology, ACR Appropriateness Criteria. Ductal Carcinoma in situ; 2014.

2. Wang L, Sulliva M, Hongyan D, et al. US Apperance of ductal carcinoma in situ. Radiographics. 2013;33(1):2130-2228.

3. American College of Radiology. Benefits and limitations of mammography. Digest of Council Action 2015-2016: 2002, amended 2012 (Res. 23-c); 2012. 44 p.

4. Hashimoto B. Sonography of ductal carcinoma in situ. Ultrasound Clinics. 2007;1(2007):631-643.

5. Takayuki Y, Naoko M, Mika W, et al. Radilogic-Pathologic Correlation of Ductal Carcinoma in Situ. Radiographics. 2010;30(5):1183-1198.

6. Kaufman S, Harris E, Bailey L, et al. Ductal Carcinoma in situ. ACR Appropriateness Criteria; 2014. 5 p.

7. Zaha D. Significance of immunohistochemistry in breast cancer. World $J$ Clin Oncol. 2014;5(3):382-392.

8. Makretsov N, Hayes M, Carter B, et al. Stromal CD10 expression in invasive breast carcinoma correlates with poor prognosis, estrogen receptor negativity, and high grade. Modern Pathology. 2007;20(1):84-89.

9. American College of Radiology practice parameter for the performance of screening and diagnostic mammography; 2013. 3 p. 